\title{
A Validated Method for the Quantitation of Ciprofloxacin Hydrochloride Using Diffuse Reflectance Infrared Fourier Transform Spectroscopy
}

\author{
Bhoomendra Bhongade, Sirajunisa Talath, and Sunil Dhaneshwar \\ Department of Pharmaceutical Chemistry, RAK College of Pharmaceutical Sciences, RAK Medical \& Health Sciences University, \\ P.O. Box 11172, Ras Al Khaimah, UAE
}

Correspondence should be addressed to Bhoomendra Bhongade; ba.bhongade@gmail.com

Received 31 October 2013; Accepted 22 December 2013; Published 5 February 2014

Academic Editor: Hakan Arslan

Copyright (C) 2014 Bhoomendra Bhongade et al. This is an open access article distributed under the Creative Commons Attribution License, which permits unrestricted use, distribution, and reproduction in any medium, provided the original work is properly cited.

\begin{abstract}
A quantitative method using diffuse reflectance infrared Fourier transform spectroscopy (DRIFTS) was developed and validated for the estimation of ciprofloxacin in its tablet dosage forms. The solid-state samples were prepared by dilution in dry potassium bromide and were analyzed by FTIR spectrophotometer with DRIFT sampling technique. A linear relationship for the carbonyl peak area centered around $1709 \mathrm{~cm}^{-1}$ was observed in the range of $0.3-1.5 \% \mathrm{w} / \mathrm{w}$ with good correlation coefficient of 0.998 . The percent recovery of ciprofloxacin in three marketed tablet dosage forms was in the range of $98.76 \pm 0.27$. The present reported method is precise, reproducible, and eco-friendly. DRIFTS may have a potential as an alternative method for qualitative and quantitative analysis of ciprofloxacin in bulk drugs and tablet dosage forms.
\end{abstract}

\section{Introduction}

Fourier transform infrared spectroscopy (FTIRS) is a widely explored technique in the pharmaceuticals and drug research for the identification of compounds, impurities, and determination of functional groups in qualitative analysis. Traditionally, FTIR analysis is carried out by transmission measurement technique using the transparent pallets of sample with halide salts. Quantification of some pharmaceutical agents has been reported in the literature using FTIR spectroscopy either by measuring the transmission of analyte in potassium bromide or in chloroform [1-4].

Because of the availability of newer sampling techniques in handling samples, FTIR is gaining more attention of the analytical researchers in the exploration and use of FTIRS technique for the quantitative analysis of solid-state samples. Diffuse reflectance infrared fourier transform (DRIFT) was proposed to be relatively better techniques for quantitative analysis of solid-state samples while attenuated total reflectance (ATR) sampling technique is more preferred method for qualitative analysis [5]. The practical difficulty that may arise for the quantification of solid-state samples using ATR and transmission measurement may be the reproducibility which can be minimized using diffuse reflectance sampling technique. The technique of DRIFT spectroscopy is concerned with the efficient collection of diffusely scattered light at the direction unrelated to that of the incident radiation. The Kubelka-Munk model described the theory of diffuse reflectance at scattering surfaces which relates band intensities to concentration for transmission measurements similar to Beer's law [6]. The literature revealed the use of DRIFTS for quantitative estimation of mixtures of sulfamethoxazole polymorphs [7], simultaneous quantitation of ethenzamide, isopropylantipyrine, caffeine, and allylisopropylacetylurea in tablet [8]. Thus, DRIFTS may have the potential to provide an efficient method for the solid-state quantification of pharmaceuticals.

The fluoroquinolones represent one of the major synthetic classes of antibacterial agents with bactericidal activity 
<smiles>O=C(O)c1cn(C2CC2)c2cc(N3CCNCC3)c(F)cc2c1=O</smiles>

FIGURE 1: Chemical structure of ciprofloxacin hydrochloride.

[9]. Ciprofloxacin hydrochloride (CAS number 85721-33-1; Figure 1), the monohydrochloride monohydrate salt of 1-cyclopropyl-6-fluoro-1,4-dihydro-4-oxo-7-(1-piperazinyl)3-quinolinecarboxylic acid indicated for the treatment of various microbial infections [10]. Fluoroquinolones interfere with the bacterial DNA gyrase resulting in the degradation of chromosomal DNA and interference with cell division and gene expression [11].

Various analytical methods the qunatitation of ciprofloxacin in bulk, pharmaceutical dosage forms and body fluids are reported. US Pharmacopoeia and Indian Pharmacopoeia describe high performance liquid chromatography (HPLC) and British Pharmacopoeia describes nonaqueous titrimetry as an official analytical methods for analysis of ciprofloxacin [12-14]. In addition, various analytical methods for the estimation of ciprofloxacin in bulk as well as pharmaceuticals including HPLC, UV-visible, and solid-state FT-Raman spectroscopy are reported and in biological fluids [15-20]. Quantitative analysis of ciprofloxacin in liquid-state by FTIR spectroscopy using phosphate buffer $\mathrm{pH} 6.0$ and methanol as a solvent was reported recently by Pande et al. [21].

To the best of our knowledge, there is no literature data published on the solvent-free analytical method for the quantification of ciprofloxacin hydrochloride in bulk and tablet dosage form using FTIR. The objectives of present study were to develop and validate the FTIR method for quantification of ciprofloxacin in solid-state and evaluate the feasibility of DRIFTS application for pharmaceuticals. The proposed DRIFTS method in the present study was performed on standard ciprofloxacin hydrochloride and three marketed tablet dosage forms, namely, Ciprowin, Ciprodac, and Omnicip tablets (Label claim $500 \mathrm{mg}$ ciprofloxacin).

\section{Materials and Methods}

2.1. Chemicals and Reagents. Standard sample of ciprofloxacin hydrochloride (Lot: 014761) was obtained as gift from Julphar-Gulf Pharmaceutical Industries, Ras Al Khaimah, UAE. Potassium bromide (Lot: 033674, analytical grade) was obtained from the HiMedia, Mumbai, India.

2.2. FTIR Instrumentation. The FTIR analyses were carried out on Cary 630 FTIR spectrophotometer equipped with diffuse reflectance sampling interface (Agilent Technologies, USA). FTIR spectra were recorded in the wavenumber range between 4000 and $650 \mathrm{~cm}^{-1}$, averaging 32 scans per sample using a nominal resolution of $8 \mathrm{~cm}^{-1}$ employing background spectrum of gold. The Cary 630 MicroLab PC software was used for data collection and Agilent Resolution Pro software was used to analyze the data.

2.3. Calibration Curve. Calibration curves were prepared for five different ciprofloxacin hydrochloride concentrations in the range of $0.3-1.5 \% \mathrm{w} / \mathrm{w}$. Appropriate quantity of ciprofloxacin hydrochloride was diluted with potassium bromide to get around $1000 \mathrm{mg}$ and triturated to ensure sample homogeneity. Each calibration standard was analyzed in the replicates of six. Area under curve (AUC) corresponding to the carbonyl peak around $1710-1702 \mathrm{~cm}^{-1}$ was used for the quantification and the average of six measurements was used to obtain the calibration curve. All the statistical calculations and calibration curve plotting were carried out using StatFlex version 6.0 software for Windows (Artech, Osaka, Japan, http://www.statflex.net/).

2.4. Method Validation. The developed DRIFTS method was validated for precision, accuracy, and linearity [22, 23].

2.4.1. Precision. The precision of method was assessed by repeatability and intermediate precision studies. Repeatability studies were performed by analyzing six samples of five different concentrations $(0.3-1.5 \% \mathrm{w} / \mathrm{w})$ of ciprofloxacin hydrochloride six times on the same day (day 1 ). The intermediate precision of assay method was evaluated by repeating studies interday (on day 3 ).

2.4.2. Accuracy. The accuracy of the assay method was evaluated by standard addition method with the recovery of pure drug from excipients at three different quantities (80, 100 , and $120 \% \mathrm{w} / \mathrm{w})$. To the preanalyzed Omnicip tablet powder, known amount of ciprofloxacin hydrochloride standard powder corresponding to 80,100 , and $120 \%$ of label claim was added. The sample was mixed thoroughly and analyzed by making appropriately $1 \% \mathrm{w} / \mathrm{w}$ dilutions with potassium bromide powder in six replicates.

2.4.3. Linearity. The linearity of calibration curve was assessed by linear regression. Solid-state samples in the concentration range of $0.5-5.0 \% \mathrm{w} / \mathrm{w}$ were prepared as described in calibration curve. Linearity of the method was studied by analyzing the six samples of five different concentrations (0.3$1.5 \% \mathrm{w} / \mathrm{w}$ ) of ciprofloxacin hydrochloride in six replicates.

2.5. Analysis of Marketed Tablet Formulations. Three different brands of ciprofloxacin tablets (Ciprowin, Ciprodac, and Omnicip; Label claim $500 \mathrm{mg}$ ciprofloxacin) were used to determine the drug content. Ten tablets were weighed accurately, their average weight determined, and finely powdered. Appropriate quantity of each tablet powder was diluted with potassium bromide powder to get around $1000 \mathrm{mg}$ sample containing approximately $1 \% \mathrm{w} / \mathrm{w}$ of ciprofloxacin hydrochloride. The solid samples were mixed thoroughly by triturating. The analysis was carried out using six samples which were analyzed in six replicates. 

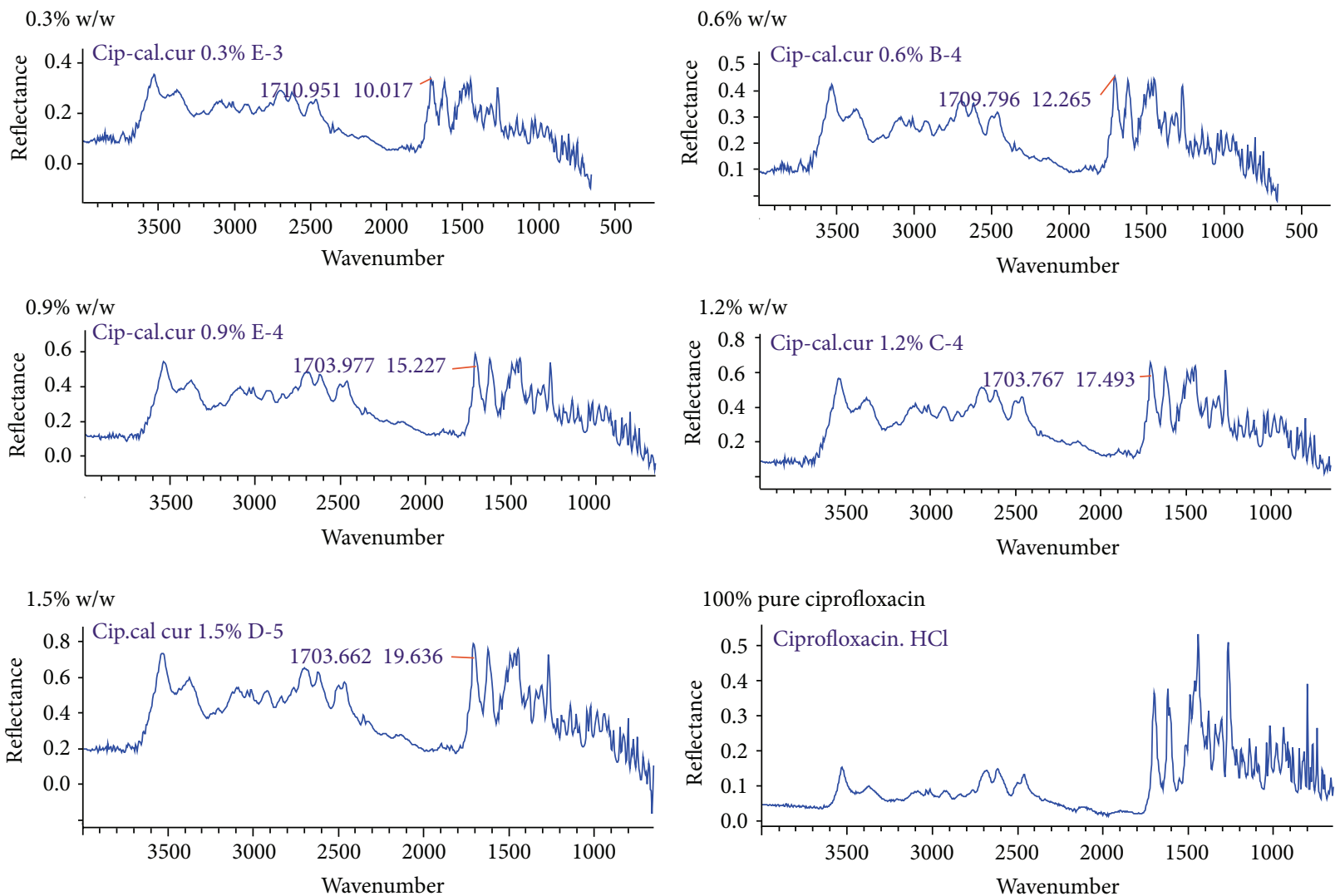

FIGURE 2: Transmittance spectra for the ciprofloxacin hydrochloride samples diluted in potassium bromide and ciprofloxacin pure drug.

\section{Results and Discussion}

Diffuse reflectance measurement of powdered samples typically results in relatively long pathlengths that increases the interaction of the infrared light with the sample. Concentrated samples may have absorbance values beyond the dynamic range of an instrument resulting in higher noise. In order to obtain the absorbance in the linear range, samples need to be diluted with nonabsorbing, diffusely reflecting salts such as potassium bromide.

The FTIR spectrum for pure sample of ciprofloxacin hydrochloride exhibited absorbance bands in the range of $3532,3373,3088,2932,2682,2620,1702,1623,1492,1446,1383$, 1342 , and $1267 \mathrm{~cm}^{-1}$. The low-intensity absorbance bands arising from ciprofloxacin were not much affected by dilution in dry potassium bromide; therefore, in the present study we have used dry potassium bromide as the diluent. The most prominent absorbance band corresponding to the carbonyl group centered in the range of $1710-1703 \mathrm{~cm}^{-1}$ for the diluted samples of ciprofloxacin in dry potassium bromide was within the 2.0 absorbance units. The transmittance spectra for the diluted ciprofloxacin samples of various concentrations are shown in Figure 2. The area under curve (AUC) for the peak centered in the range of $1710-1703 \mathrm{~cm}^{-1}$ was used for the preparation of calibration curve as shown in Figure 3. The
TABLE 1: Linear regression data for ciprofloxacin hydrochloride.

\begin{tabular}{lc}
\hline Statistical parameters & Values \\
\hline Concentration range & $0.3-1.5 \% \mathrm{w} / \mathrm{w}$ \\
Regression equation & $y=-0.9418+0.12708 x$ \\
Correlation coefficient & 0.998 \\
\hline
\end{tabular}

calibration curve is described by the equation $y=a+b x$, where $y$ represents peak area and $x$ represents concentration of ciprofloxacin hydrochloride. Initially the samples in the concentration range of $0.5-5.0 \% \mathrm{w} / \mathrm{w}$ were analyzed to determine the linearity. The calibration curve with good linearity was established ranging from 0.3 to $1.5 \% \mathrm{w} / \mathrm{w}$ ciprofloxacin in potassium bromide. The corresponding linear regression equation was $y=-0.9418+0.12708 x$ and the correlation coefficient for calibration curve was 0.998 (Figure 3 and Table 1).

The precision was expressed by coefficient of variation (\%RSD) and accuracy by mean and standard deviation. For day 1 precision studies, the RSD (\%) values for the five samples was observed in the range of 0.837-1.707 while for day 3 precision studies the range was $0.660-2.428$ (Table 2). 
TABLE 2: Intraday and interday precision and accuracy for analysis data of ciprofloxacin hydrochloride $(n=6)$.

\begin{tabular}{|c|c|c|c|c|c|c|}
\hline \multirow{2}{*}{ Nominal concentration $(\% \mathrm{w} / \mathrm{w})$} & \multicolumn{3}{|c|}{ Day 1} & \multicolumn{3}{|c|}{ Day 3} \\
\hline & AUC & SD & RSD (\%) & AUC & SD & $\operatorname{RSD}(\%)$ \\
\hline 0.278 & 9.431 & 0.160 & 1.707 & 8.702 & 0.212 & 2.428 \\
\hline 0.612 & 12.434 & 0.166 & 1.341 & 12.280 & 0.157 & 1.282 \\
\hline 0.963 & 15.227 & 0.151 & 0.881 & 15.548 & 0.216 & 1.388 \\
\hline 1.256 & 16.975 & 0.186 & 0.951 & 17.317 & 0.113 & 0.660 \\
\hline 1.495 & 19.217 & 0.160 & 0.837 & 19.376 & 0.129 & 0.666 \\
\hline
\end{tabular}

TABLE 3: Recovery data of ciprofloxacin in tablet $(n=6)$.

\begin{tabular}{|c|c|c|c|c|c|c|c|}
\hline Tablet brand name & Label claim (mg) & Amount of drug added (mg) & Total & Amount recovered (mg) & \% Recovery & $\mathrm{SD}$ & RSD (\%) \\
\hline \multirow{3}{*}{ Omnicip } & 500 & 400 & 900 & 933.79 & 103.75 & 3.74 & 3.61 \\
\hline & 500 & 500 & 1000 & 1021.2 & 102.12 & 5.654 & 5.56 \\
\hline & 500 & 600 & 1100 & 1084.43 & 98.585 & 3.545 & 3.59 \\
\hline
\end{tabular}

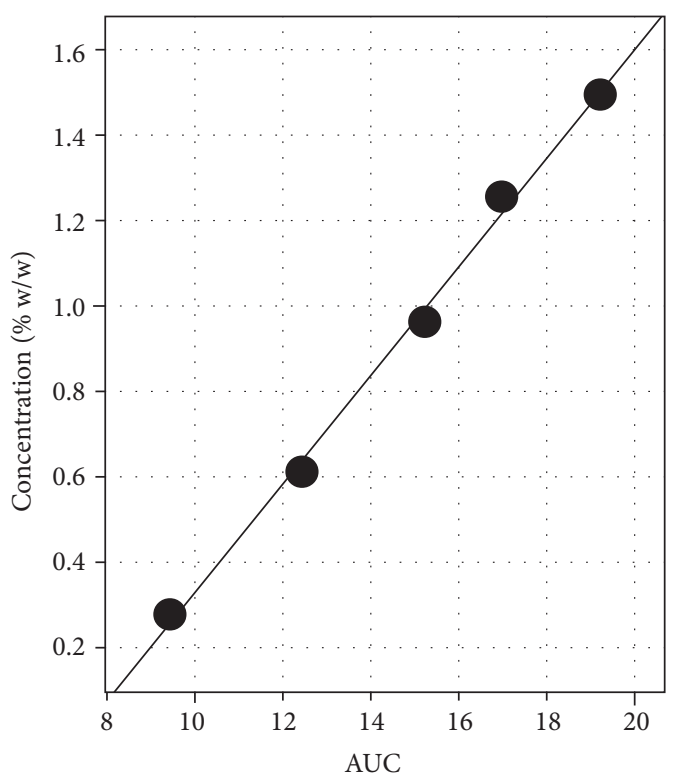

Figure 3: Calibration curve for ciprofloxacin hydrochloride $(0.3-$ $1.5 \% \mathrm{w} / \mathrm{w})$.

The intraday and interday precision results were within the accepted variable limits.

The accuracy of the assay method was evaluated with the recovery of pure drug from excipients at three different levels $(80,100$, and $120 \% \mathrm{w} / \mathrm{w}$ of label claim) by standard addition method and the recovery data is summarized in Table 3. Good recoveries of ciprofloxacin hydrochloride were obtained in the range of $98.58-103.75 \%$ with the RSD range of 3.51-5.56 at various added concentrations.
The proposed validated method was applied for the quantification of ciprofloxacin hydrochloride in tablet dosage form. The FTIR spectra for the representative samples of tablet dosage forms diluted with potassium bromide are shown in Figure 4 . The FTIR spectrum in Figure 4 indicates that there is no interference of excipients used in the formulation of tablet dosage form. Three different brands of ciprofloxacin tablets were analyzed using the developed method and the results of analysis are shown in Table 4. The average recoveries of ciprofloxacin hydrochloride in all the three formulations were in the range of $98.46-98.99 \% \mathrm{w} / \mathrm{w}$ of label claim and the \%RSD values were in the range of $4.77-$ 5.05. The \%recovery of label claim was in good agreement and within the acceptable limits of the USP (not less than $90.0 \%$ and not more than $110.0 \%$ of the stated amount of ciprofloxacin).

\section{Conclusion}

Traditionally, FTIR spectroscopy is employed for the qualitative analysis of pharmaceuticals; however, with advent in sampling techniques, DRIFT spectroscopy may serve as useful technique for qualitative and quantitative analysis of solid-state pharmaceuticals. In the present paper, we report the development and validation of eco-friendly DRIFTS method for the quantification of solid-state ciprofloxacin hydrochloride and its successful application to pharmaceuticals. The proposed method was found to be precise, accurate, and suitable for analysis of ciprofloxacin as bulk drug and in pharmaceutical formulation. Thus, the developed method has the advantage of being solvent free, eco-friendly, and cost effective and involving relatively simple sample preparation. The developed validated method can be useful for the routine quality control analysis of ciprofloxacin in pharmaceuticals industries with desired precision and accuracy. 
TABLE 4: Assay results of tablets $(n=6)$.

\begin{tabular}{|c|c|c|c|c|c|}
\hline Tablet brand names & Label claim (mg) & Amount recovered (mg) & \% Recovery & SD & RSD (\%) \\
\hline Ciprowin & 500 & 492.30 & 98.46 & 4.69 & 4.772 \\
\hline Ciprodac & 500 & 494.20 & 98.841 & 6.578 & 6.655 \\
\hline Omnicip & 500 & 494.96 & 98.992 & 5.00 & 5.05 \\
\hline
\end{tabular}
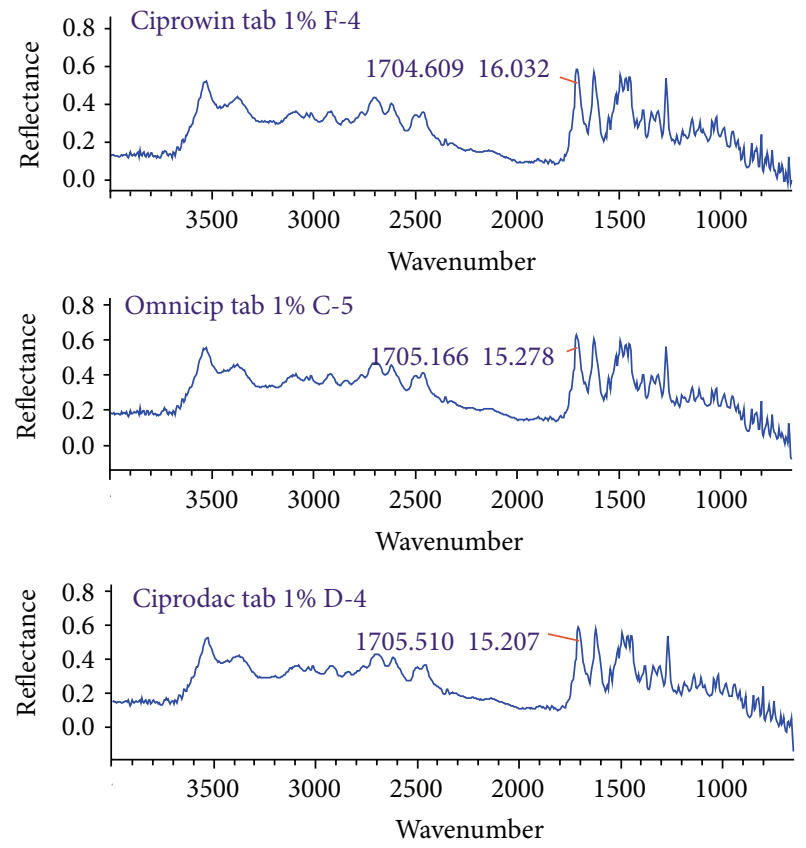

FIGURE 4: Transmittance spectra for the marketed tablet powder diluted in potassium bromide.

\section{Conflict of Interests}

The authors declare that there is no conflict of interests in the publication of the paper.

\section{Acknowledgments}

The authors thank Dr. B. G. Nagavi, Dean, RAKCOPS; Dr. S. Gurumadhva Rao, Vice Chancellor, RAKMHSU, for their encouragement and providing facilities. They also thank Julphar-Gulf Pharmaceutical Industries, Ras Al Khaimah, UAE, for providing the gift sample of standard ciprofloxacin hydrochloride.

\section{References}

[1] R. Bansal, A. Guleria, and P. C. Acharya, "FT-IR method development and validation for quantitative estimation of ziduvudine in bulk and tablet dosage form," Drug Research, vol. 63, pp. 165-170, 2013.

[2] E. Konoz, A. H. M. Sarrafi, M. Samadizadeh, and S. Boreiri, "Quantitative analysis of lorazepam in pharmaceutical formulation through FTIR spectroscopy," E-Journal of Chemistry, vol. 9, pp. 2232-2238, 2012.
[3] K. C. Patraa, K. J. Kumarb, S. K. Sahooa, and P. Suresha, "Rapid FTIR method for estimation of sucrose in a traditional indian polyherbal formulation," Eurasian Journal of Analytical Chemistry, vol. 5, pp. 73-80, 2010.

[4] S. R. Matkovic, G. M. Valle, and L. E. Briand, "Quantitative analysis of ibuprofen in pharmaceutical formulations through FTIR spectroscopy," Latin American Applied Research, vol. 25, pp. 189-195, 2005.

[5] F. Higgins and J. Seelenbinder, "Quantitative measurement of active pharmaceutical ingredients using the diffuse reflectance Cary 630 FTIR," Agilent technologies, Danbury, Conn, USA, Application note, Publication number: 5990-9414EN, 2011, http://www.chem.agilent.com/Library/applications/59909414EN_AppNote_630_ActiveIngredients.pdf.

[6] P. Kubelka and F. Munk, "Ein Beitrag zur Optik der. Farbanstriche," Zeitschrift Tech Physikalische, vol. 12, pp. 593-595, 1931.

[7] K. J. Hartauer, E. S. Miller, and J. K. Guillory, "Diffuse reflectance infrared Fourier transform spectroscopy for the quantitative analysis of mixtures of polymorphs," International Journal of Pharmaceutics, vol. 85, no. 1-3, pp. 163-174, 1992.

[8] M. Park, H. Yoon, K. Kim, and J. Cho, "Quantitative analysis by diffuse reflectance infrared Fourier transform and linear stepwise multiple regression analysis I -Simultaneous quantitation of ethenzamide, isopropylantipyrine, caffeine, and allylisopropylacetylurea in tablet by DRIFT and linear stepwise multiple regression analysis-," Archives of Pharmacal Research, vol. 11, no. 2, pp. 99-113, 1988.

[9] M. V. N. de Souza, "New fluoroquinolones: a class of potent antibiotics," Mini-Reviews in Medicinal Chemistry, vol. 5, no. 11, pp. 1009-1017, 2005.

[10] M. LeBel, "Ciprofloxacin: chemistry, mechanism of action, resistance, antimicrobial spectrum, pharmacokinetics, clinical trials, and adverse reactions," Pharmacotherapy, vol. 8, no. 1, pp. 3-33, 1988.

[11] D. C. Hooper, "Mechanisms of action of antimicrobials: focus on fluoroquinolones," Clinical Infectious Diseases, vol. 32, no. 1, pp. S9-S15, 2001.

[12] The United States Pharmacopoeia, USP 29-NF 24, the United States Pharmacopoeial Convention, Rockville, Md, USA, 2008.

[13] Indian Pharmacopoeia, Ministry of Health \& Family Welfare, Pharmacopoeia Commission, Ghaziabad, India, 6th edition, 2007.

[14] British Pharmacopoeia, Her Majesty's Stationary Office, London, UK, 2000.

[15] M. A. Al Omar, "Ciprofloxacin," in Profiles of Drug Substances, Excipients, and Related Methodology, vol. 31, pp. 163-214, Academic Press, Brittain, Va, USA, 2004.

[16] G. Carlucci, "Analysis of fluoroquinolones in biological fluids by high- performance liquid chromatography," Journal of Chromatography A, vol. 812, no. 1-2, pp. 343-367, 1998.

[17] A. Dincel, A. Yildirim, F. Caglayan, and A. Bozkurt, "Determination of ciprofloxacin in human gingival crevicular fluid 
by high-performance liquid chromatography," Acta Chromatographica, no. 15, pp. 308-314, 2005.

[18] S.-S. Wu, C.-Y. Chein, and Y.-H. Wen, "Analysis of ciprofloxacin by a simple high-performance liquid chromatography method," Journal of Chromatographic Science, vol. 46, no. 6, pp. 490-495, 2008.

[19] I. M. van Geijlswijk, A. R. H. van Zanten, and Y. Geert van der Meer, "Reliable new high-performance liquid chromatographic method for the determination of ciprofloxacin in human serum," Therapeutic Drug Monitoring, vol. 28, no. 2, pp. 278-281, 2006.

[20] Z. Vybíralová, M. Nobilis, J. Zoulova, J. Květina, and P. Petr, "High-performance liquid chromatographic determination of ciprofloxacin in plasma samples," Journal of Pharmaceutical and Biomedical Analysis, vol. 37, no. 5, pp. 851-858, 2005.

[21] S. Pande, P. Pandey, G. Tiwari, R. Tiwari, and A. K. Rai, "FTIR spectroscopy: a tool for quantitative analysis of ciprofloxacin in tablets," Indian Journal of Pharmaceutical Sciences, vol. 74, pp. 86-90, 2012.

[22] ICH Quality Guidelines Q2A, Text on Validation of Analytical Procedures, Q2B, Validation of Analytical Procedures: Methodology, Geneva, Switzerland, 1996.

[23] Guidelines for the Development and Validation of Near Infrared (NIR) Spectroscopic Methods, Pharmaceutical Science Group, NIR sub group, UK, 2001, http://www.pasg.org.uk/ NIR/NIR_Guidelines_Oct_01.pdf. 

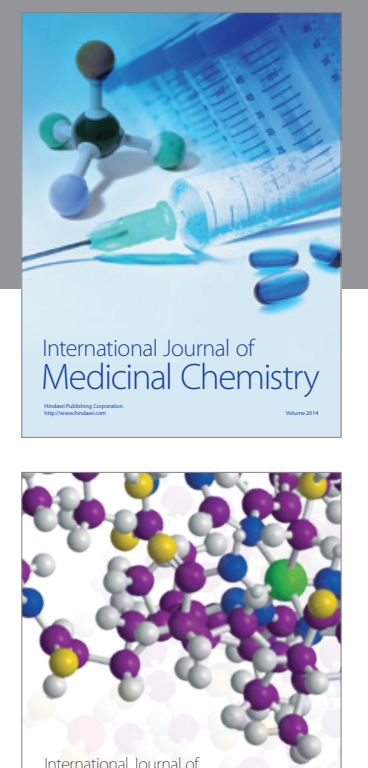

\section{Carbohydrate} Chemistry

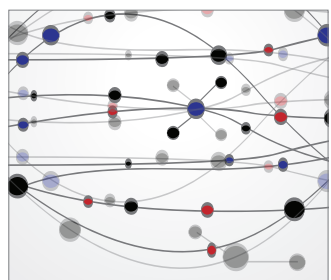

The Scientific World Journal
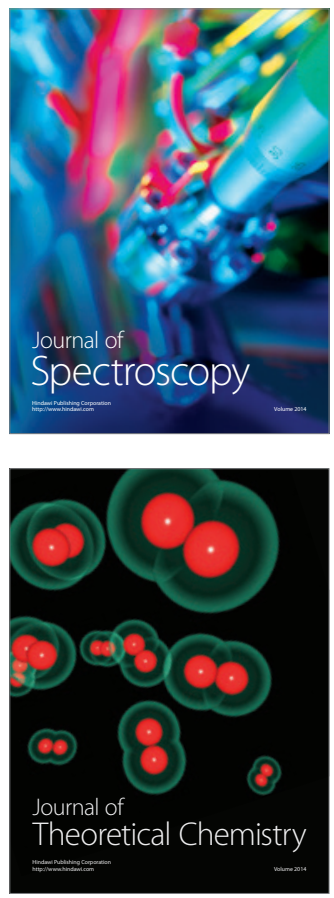
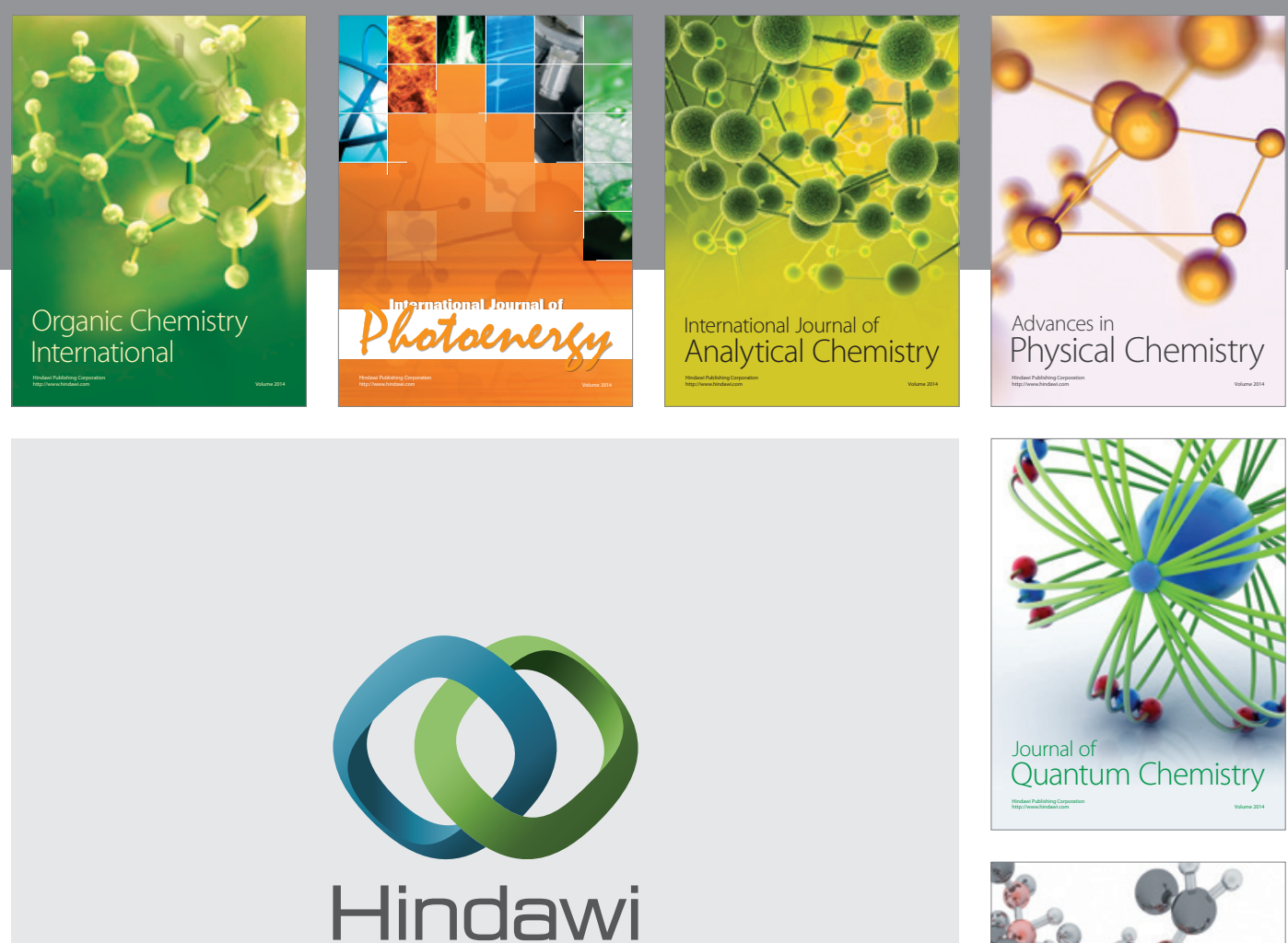

Submit your manuscripts at

http://www.hindawi.com

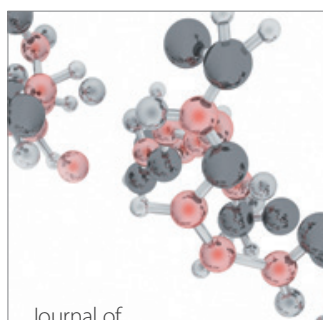

Analytical Methods

in Chemistry

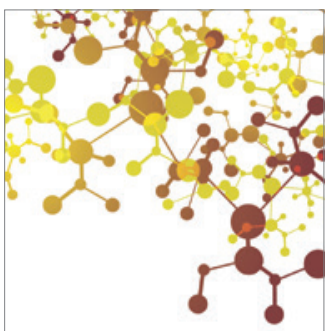

Journal of

Applied Chemistry

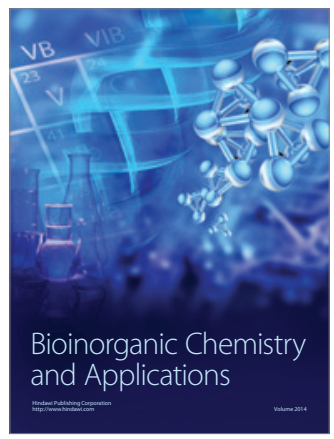

Inorganic Chemistry
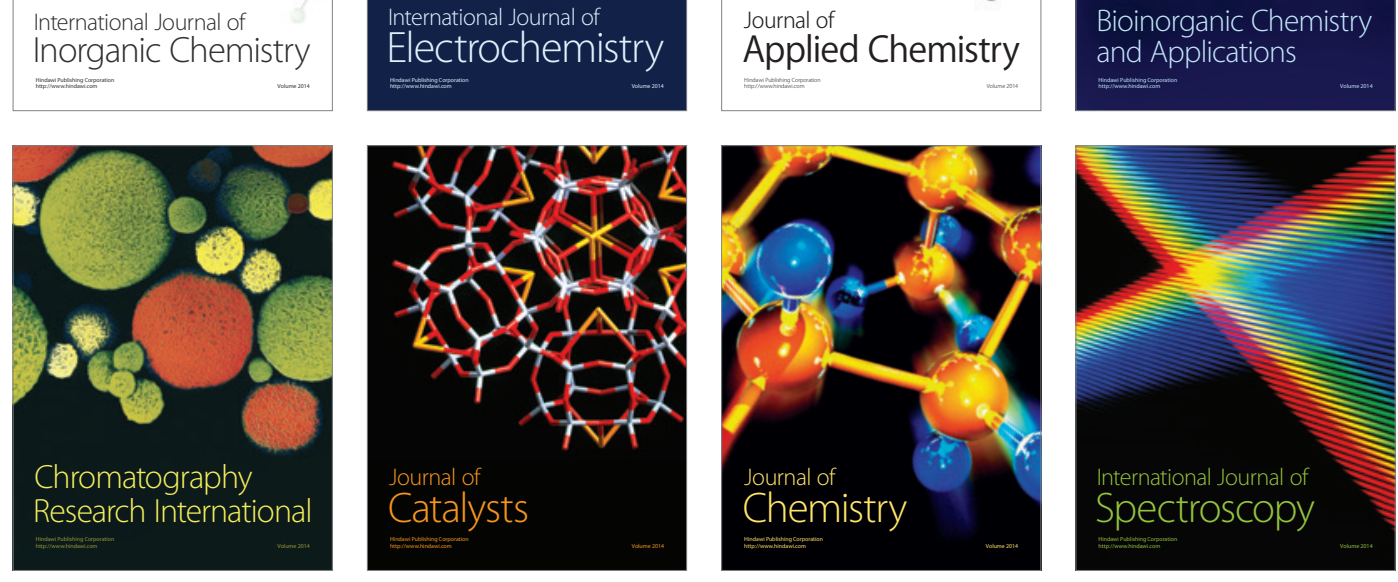